Fordham University

Fordham Research Commons

\title{
"That's the Beauty of it": Practitioners Describe the Affordances of Direct to Consumer Tele-Mental Health
}

\author{
Lauri Goldkind
}

Lea Wolf

CUNY Grad Center

Follow this and additional works at: https://research.library.fordham.edu/gss_facultypubs

Part of the Counselor Education Commons, Other Psychology Commons, Social Justice Commons, and the Social Work Commons

\section{Recommended Citation}

Goldkind, Lauri and Wolf, Lea, "'That's the Beauty of it": Practitioners Describe the Affordances of Direct to Consumer Tele-Mental Health" (2021). Social Service Faculty Publications. 20.

https://research.library.fordham.edu/gss_facultypubs/20

This Article is brought to you for free and open access by the Graduate School of Social Service at Fordham Research Commons. It has been accepted for inclusion in Social Service Faculty Publications by an authorized administrator of Fordham Research Commons. For more information, please contact considine@fordham.edu, bkilee@fordham.edu. 


\section{"That's the Beauty of it": \\ Practitioners Describe the \\ Affordances of Direct to \\ Consumer Tele-Mental Health}

Families in Society: The Journal of

Contemporary Social Services

I-16

(C) The Author(s) 2021

Article reuse guidelines:

sagepub.com/journals-permissions DOI: I0.1 I77/I04438942/997796 journals.sagepub.com/home/fis

๑SAGE

\author{
Lauri Goldkind' (iD) and Lea Wolf ${ }^{2}$
}

\begin{abstract}
Tele-mental health, or the provision of remote counseling services, has been available for decades. This qualitative study uses the framework of affordances, derived from Gibson, to examine what social work practitioners working on direct to consumer tele-mental health (DTCTMH) platforms are discovering about the features, benefits, and constraints of virtual therapy. An interpretive phenomenological approach was employed to document the lived experiences of social workers who practice in this manner. According to the practitioners interviewed, for a subset of individuals seeking treatment, DTCTMH can offer meaningful interpersonal interaction that confers benefit. Key affordances include accessibility, anonymity, meaningful work, autonomy, lifelong learning, and access by new populations. Practitioners simultaneously acknowledge the ethical complexities and structural challenges of DTCTMH practice. The article concludes with suggestions for future research, policy, and practice.
\end{abstract}

\title{
Keywords
}

affordances, tele-mental health, clinical practice, digital service delivery, online therapy, e-therapy, direct to consumer tele-mental health

Manuscript received: October 13, 2020; Revised: December 29, 2020; Accepted: January 21, 2021

Disposition editor: Sondra J. Fogel

\section{Introduction}

In the United States, the need for mental health services is unmatched by the supply of providers. A 2017 study from the Substance Abuse and Mental Health Services Administration found that of the 46.6 million Americans with a mental health concern, fewer than half $(42.6 \%)$ received treatment (Mental Illness, 2019). Reform advocates and major institutional providers, including the federal government, have endorsed digital mental health interventions as a solution to persistent problems within health care, as a tool to cut costs and to increase the supply of evidencebased treatment available (Barak \& Grohol,
2011). For those seeking accessible mental health treatment, tele-mental health (TMH) offers virtual access to licensed practitioners, whereas for social workers committed to clinical practice, various TMH configurations can offer a flexible and autonomous opportunity

\footnotetext{
'PhD, associate professor, Fordham University, New York City, NY, USA

${ }^{2}$ LMSW, doctoral student, The City University of New York, New York City, USA

Corresponding Author:

Lauri Goldkind, Graduate School of Social Service,

Fordham University, II 3 West 60th Street, New York, NY 10023, USA.

Email: goldkind@fordham.edu
} 
for meaningful work outside of agency settings. Yet few master's-level social work courses teach the skills required to practice in digital spaces (Goldkind, 2020), despite the growing need for professionals to understand the ethical, relational, and practical dimensions of providing mental health services in an accelerating technological culture.

TMH includes synchronous and asynchronous electronic communications between a client and a social worker aimed at improving an individual's mental and behavioral health (Colbow, 2013). An expanding body of research documents positive outcomes for TMH across conditions and populations, when conducted synchronously using telephone or videoconference (Andrews et al., 2010; Karyotaki et al., 2017; Turgoose et al., 2018; Varker et al., 2019). A growing body of literature describes a multiverse of technologies that can be usefully incorporated into mental health treatment, including asynchronous and mobile options (Hilty et al., 2013). Chan et al. (2018) argue that asynchronous modes are best deployed to augment and extend treatment, and research documents efficacy. A 2016 review of 36 studies, text messaging was successful in providing reminders, information, and supportive messaging to patients while promoting self-monitoring (Berrouiguet et al., 2016) and text messaging has been documented to provide effective reach to marginalized groups and populations with low digital literacy (Figueroa \& Aguilera, 2020).

There is growing evidence for the effectiveness of mental health treatment and support provided by a range of digital mental health channels including mobile apps (Firth et al., 2017; Neary \& Schueller, 2018). Research has yet to explore the overlap of TMH with novel direct to consumer tele-mental health (DTCTMH) models, or the provision of therapy via digital "matching" services, similar to those popularized for ridesharing, household chores, and grocery shopping. What does such intervention look like; what compels clinicians to offer their services via platform; and how do they understand the work they do with clients via platform? Literature has begun to interrogate practitioner's perspective, making the association between provider attitudes and the uptake/spread of new treatment paradigms (Békés \& Aafjes-van Doorn, 2020; Glueckauf et al., 2018); however, this lens has yet to be extended to social workers. To fill this gap, the authors focused on social workers serving clients on privately operated TMH platforms in an arrangement referred to as DTCTMH, using the lens of affordances to explore the unique potentials of this modality. TMH has long been assessed by comparison with faceto-face therapy, yet its virtual form affords distinct use and possibility. As one practitioner offered,

I think there's value in it . . . . I don't think faceto-face will ever go away, I think there's value, and I think it's just another modality for deliverance, you know. It's not to replace, it's to enhance.

Using the lived experiences of professional therapists, this study provides a perspective on how social workers experience virtual practice in the specific spaces of platformbased therapy.

\section{Literature Review}

\section{$\mathrm{TMH}$}

$\mathrm{TMH}$ is the delivery of mental health services using information and communication technologies. Many versions of TMH delivery have been in use since at least the 1970s. These include traditional telephone and video-conferencing, equipment for synchronous (realtime) communication, as well as asynchronous (store and forward) technologies, such as email or proprietary platforms, which allow practitioners to conduct therapeutic interchange (Luxton et al., 2014; Vernig, 2016).

Discussion of virtual therapeutic interchange has long centered on questions of relative efficacy and of ethical challenges. A growing body of research documents the therapeutic utility of virtual interventions to promote mental health and well-being. A 2017 National Institutes of Health (NIH)-funded 
systematic review found evidence that TMH can provide effective interventions to patients with multiple conditions-including depression, dementia, schizophrenia, and panic disorders - and that the modality offers meaningful potential to child psychiatry (Langarizadeh et al., 2017). Another review suggests evidence for the efficacy of mobile apps in reducing depression, anxiety, stress, and possibly substance use (Rebello et al., 2014). Furthermore, TMH provides access to effective mental health services to populations who are otherwise unable or unlikely to seek care (Godleski et al., 2012), including those who live in isolated communities (Langarizadeh et al., 2017). A majority of studies examining TMH interventions conclude with calls for further research, and questions about the efficacy of different communication channels, the parameters of client benefit, and appropriate dosage and duration of treatment require further study.

Although existing codes - the National Association of Social Workers (NASW) Code of Ethics and the NASW, ASWB, CSWE, and CSWA Standards for Technology in Social Work Practice-address the principles that guide ethical practice in virtual space, TMH generates novel treatment configurations and dilemmas. Research suggests that some components of therapy, such as securing informed consent, may be rendered complex by the context of TMH (Goldkind \& Wolf, 2020; Malhotra et al., 2013; Sabin \& Skimming, 2015). Practitioners confirm that, across virtual modalities, many of the primary protocols of mental health treatment-from contracting to safety planning to the promise of confidentialityrequire active reimagining (Goldkind \& Wolf, 2020). It has been noted that clinicians continue to have mixed views when asked to deliver TMH services (Payne et al., 2020; Trub \& Magaldi, 2017), and that practitioners feel individually responsible to negotiate the ethics of online practice, including confidentiality and the ability to manage crisis remotely (Glueckauf et al., 2018). Some literature finds that clients are more comfortable with TMH than are practitioners (Watts et al., 2020), though use of virtual modalities encourages their endorsement by therapists (Békés \& Aafjes-van Doorn, 2020). In the research literature, practitioners continue to debate what is lost when therapist and patient are not face-to-face, including the resource of nonverbal forms of communication (HaigFerguson et al., 2019), while asserting the need for professional training specific to virtual modalities (Wilkerson et al., 2020).

\section{DTCTMH}

Technology has facilitated new forms of social and economic interaction, including the platforms explored in this study. DTCTMH is a model for delivering mental health services whereby a third-party provider (referred to as a "platform") serves as a broker and/or matchmaking service between consumers and mental health professionals. The platform provides a digital dashboard, a suite of tools that allows clients to review potential providers, and offers a proprietary channel for subsequent communications. Clients and therapists communicate exclusively via the platform-provided channels, and the platform's digital infrastructure allows it to assume administrative functions: keeping records, nudging therapists to respond to clients within a designated time window, billing clients, and calculating therapist remuneration. The DTCTMH model has been used to deliver mental health services for nearly a decade (Goldkind \& Wolf, 2020), allowing private sector, for-profit suppliers, including Breakthrough, BetterHelp, iTherapy and Talkspace, and others, to offer "on-demand" virtual mental health services to clients across state and even national boundaries. Most DTCTMH platforms offer a range of communication options to choose from synchronous video conference or telephone, asynchronous text messaging or emails, or a hybrid. Both practitioners and clients can elect the mechanism(s) by which they connect (Goldkind \& Wolf, 2020; Payne et al., 2020).

In this model of mental health service delivery, it is up to a client-consumer to assess a platform's quality of service, to research how each provider vets contractors, to locate a policy on privacy, and to figure out which 
admixture of therapeutic modalities might be most optimal for them (Trub \& Magaldi, 2017). Given the lack of regulation of TMH platforms at the federal and state levels, social workers who contract with these platforms also face enhanced responsibility to practice in accordance with their licensure.

\section{Affordance as a Theoretical Framework}

In the late 1960s, American psychologist James Jerome Gibson introduced the concept of affordances to explain how the raw physics of vision become a meaningful interpretation of the world. Gibson (2015) wrote "the affordances of the environment are what it offers the animal" (p. 119), defining an affordance as the potential for action that is generated by the relationship between a specific animal and a particular object or environs. Affordances, in Gibson's model, are the means by which environmental stimuli are metabolized to meaning. As Bucher and Helmond (2016) explain, "we do not perceive the environment as such, but rather perceive it through its affordances, the possibilities for action it may provide" ( $p$. 2 ); in other words, humans perceive a chair in terms of the actions and experiences it offers to them-how it enables sitting, or taking a rest. Yet affordances are specific relationships, dependent on who is perceiving, and the unique abilities of the individual. A box might have the affordance of transporting objects for an adult who is strong enough to carry it when full, but may afford a toy house for a child who is too small to perform the same action. For a digital native, a laptop affords connection, whereas the same machine may be inert - affording nothing - in the hands of a person without technological skills. Affordances and their opposite, constraints, map roughly onto possibilities for/barriers to action. A laptop offers possibilities for (affords) communication, whereas poor broadband is a barrier to (constrain) those actions. The affordance framework has proven a generative model for exploring effective human-object interaction in disciplines from psychology to technology.
Gibson's affordances have many conceptual offspring. "Technology affordances," first articulated by Gaver (1991), initially expanded the design imperative of maximizing affordance into design of hardware and software. Later iterations focus on how the material qualities of technology afford "sociality and communicative actions" (Bucher \& Helmond, 2016, p. 21). Social affordances are defined as "the social structures that take shape in association with a given technical structure" (Postigo, 2016, in Bucher \& Helmond, 2016, p. 9), for example, the ways in which the internet shapes collective experience (Wellman et al., 2003). This study incorporates these evolving definitions as useful tools, centering discussion on how platforms that facilitate mental health make specific actions or experiences available to their users. The findings below distill interviews with practitioners to isolate what Hutchby (2001) labels "communicative affordances," or the "possibilities for action that emerge from ... given technological forms" (p. 30).

\section{Study Questions}

In this study, the authors asked practitioners to describe their lived experience of providing DTCTMH services. This study explores two questions:

1. How do social workers describe the experience of practicing TMH on a direct to consumer platform?

2. What actions or experiences does DTCTMH afford to clients and practitioners?

\section{Method}

This qualitative study used an interpretative phenomenological analysis (IPA) to guide the analysis of interview data from social work practitioners. IPA focuses on how people understand and make sense of a particular phenomenon, seeking to explore both the unique and common elements of their experience (Smith et al., 2009). The rationale for using IPA in this study is its suitability for 
exploratory research, as it focuses on how people understand and make sense of their experiences (Hood, 2015).

The study was conducted under the auspices of the authors' Institutional Review Board (IRB). An interview protocol was developed by the two authors in consultation with a licensed clinical social worker who had training and practice experience in TMH. Interview questions were organized under the broad umbrella of TMH operations: entry into $\mathrm{TMH}$, training, client assignment, clinical assessment, compensation, and supervision. Practitioners were also asked to reflect on the unique affordances and constraints of their practice and to describe its ethical dimensions. All of the participants were practicing, or had practiced on, one or more privately operated TMH platforms.

\section{Sample}

Participants were recruited from the websites of two major national private, for-profit DTCTMH platforms. These platforms were selected as they are national in scope and provide the credentials and names of providers publicly. A total of 750 licensed clinical master of social work practitioners received postcard invitations for an initial interview, followed by two reminder follow-up mailings. A total of 50 individuals agreed to participate in an interview, 30 interview appointments were scheduled, and 21 individual telephone interviews were ultimately conducted. Interviews were audio-recorded and transcribed by a professional transcriptionist and imported into the qualitative data analysis software Dedoose.

Table 1 summarizes the demographic details of the study's participants. Participants ranged in age from 26 to above 71 , with most of the practitioners being 45 to $55(n=9)$. Seven of the participants were practicing TMH on two or more platforms. Social workers in this sample are consistent with other demographic reports surveying the field (Salsberg et al., 2017). The sample was predominately female $(n=19)$ and majority White $(n=14)$ but did include three Black and four Latinx social workers.

\section{Data Analysis}

Interviews were audio-recorded with participant consent. Transcripts were analyzed in successive stages (Smith et al., 2009), starting with immersion in the data through reading and repeated listening to the audio recordings. The transcripts were coded using techniques suggested by Corbin and Strauss (1990). This process included independent reading and coding of the transcripts to identify codes, comparison between investigators, and refinement of conceptual categories via consensus-building discussion (Corbin \& Strauss, 1990). In the open-coding phase of data preparation, more than 40 unique codes were generated by the authors. After the coding became redundant, authors met and compared their results (Padgett, 1998), generating themes out of the language participants used to describe their lived experience. As Merolli et al. (2014) point out, in the realm of phenomenological research, a secondary thematic analysis may assist the researchers in identifying meaning within themes to further contextualize the findings. In this case, the researchers arranged the themes as affordances, providing a frame in which findings can be viewed from the participant's perspective, in their own words.

To increase the validity of the findings, focus groups were convened for a memberchecking process (Birt et al., 2016), in which participants respond to an initial synthesis of findings, co-constructing the final set. Six practitioners participated in these sessions, providing feedback and discussion on the themes and findings resulting from the analysis of data.

\section{Findings and Discussion}

The findings below speak to affordances which describe the action, social structures, and communication potentials that evolve alongside technology and technological systems like the platforms under study. These affordances are those that a specific individual, group of users, or organization can execute by using the properties of a technology or information system (Hutchby, 2001). Affordances, 
Table I. Demographic Characteristics $(N=21)$.

\begin{tabular}{lr}
\hline Age & \\
26 to 30 & I \\
31 to 35 & 4 \\
36 to 40 & 4 \\
41 to 45 & 2 \\
46 to 50 & 6 \\
51 to 55 & 3 \\
Above 7 I & 1 \\
Race & \\
African American & 3 \\
Latinx & 4 \\
White & 14 \\
Gender & \\
Female & 19 \\
Male & 2 \\
Geographic region & \\
Midwest & 5 \\
Northeast & 11 \\
Southwest \\
West & 4 \\
No. of platforms & 1 \\
0-I & \\
2-3 & \\
3 or more & 44 \\
Year masters conferred & 4 \\
1990-1999 & 3 \\
2000-2009 & \\
\hline
\end{tabular}

and their paired inverse, constraints, are each a relational concept, specific potential interactions between people and technology, rather than fixed properties of either users or technology (Gaver, 1996). The analysis below first outlines two primary affordances that are relevant to both practitioners and clientsaccessibility and anonymity. It continues to describe how DTCTMH affords practitioners meaningful work, autonomy, lifelong learning, and reach to new populations. Table 2 summarizes the findings.

\section{Accessibility}

As marketed by the platforms, DTCTMH is accessible and immediate, an always-on conduit to empathetic response: ads for the services use taglines like "Message your therapist anytime from anywhere." The process of signing up is designed to minimize friction: a series of lucid graphics guides a client through an account setup process, an opaque term of service agreement, and a brief automated assessment. Even when their reactions to the modality are complex or fraught, practitioners endorse the accessibility of platform-based therapy, testifying that platforms offer clients the ability to engage in a supportive therapeutic relationship which centers individual choice: the ability to select from an array of available practitioners, to elect their preferred means of communication, and to determine the frequency of contact. As one practitioner said, "I think it's incredibly user-friendly." Another described how platform technology affords a practice which foregrounds client control of treatment parameters: "it's client-centered, so the client wants a tenminute interaction that's what they get and that's what you get paid for. And if the client wants a ninety-minute intervention that's what they get." Some practitioners see the technologically facilitated "custom" nature of the modality as a mutual benefit, as it correlates client need to therapeutic contact: "So I think the great virtue of it is that it's 'as needed,' and so the client is highly motivated every time they contact you." Therapists describe accessibility as an affordance that goes deeper than merely catering to human impatience: one practitioner cited "the immediacy to access of care" as both an answer to client need and an ethical potential of DTCTMH.

Platform-based therapy is additionally accessible to clients also because it provides contact with licensed professionals at lower cost to clients than traditional face-to-face therapy. As one practitioner states, "people who don't have insurance, you know it's a reasonable cost in order to receive therapy, where they might not be able to pay the outof-pocket expenses sitting in somebody's office every week." Plans at the two largest platforms range from US $\$ 35$ per week to US\$79 per week, depending on the services offered and the amount of time to which a client commits. Providing therapy via platform is also less expensive for a therapist, eliminating the costs of space rental, advertising, and the administrative expenses of billing and record keeping. Beyond cost, 
Table 2. Affordance Key Findings.

\begin{tabular}{|c|c|}
\hline Actor & Affordance \\
\hline Consumer/client & $\begin{array}{l}\text { - Autonomy via choice of therapeutic modality, session frequency and length of } \\
\text { treatment } \\
\text { - Expanded access to providers } \\
\text { - Flexibility to schedule around one's needs } \\
\text { - Cost is accessible to those who may not have mental health insurance coverage } \\
\text { - Anonymity offers those who might otherwise not seek services (including those } \\
\text { whose behavior sets them outside of societal norms) a pathway to mental } \\
\text { health services }\end{array}$ \\
\hline Practitioner & $\begin{array}{l}\text { - Autonomy to craft one's professional life, either in the form of a private } \\
\text { practice or supplementing and existing traditional job } \\
\text { - Flexibility to schedule around one's needs } \\
\text { - Friction created by health insurance networks and health insurance provider } \\
\text { billing structures removed } \\
\text { - Meaningul work } \\
\text { - Extended reach: serve new clients and populations } \\
\text { - Lifelong learning: opportunity to cultivate new skills }\end{array}$ \\
\hline
\end{tabular}

face-to-face therapy can be resource-intensive for clients, and platforms streamline and simplify the arc of treatment: appearing to prescreen providers, removing travel time, and eliminating the stricture of the $50-\mathrm{min}$ hour. Virtual therapy, available by screen or telephone, extends access to "people that live in remote places that can't get access to care. People that are medically incapable of leaving the home, you know, elderly. I just think people that might have more obstacles to reach someone." Many identify clients who are physically or psychologically unable to travel, including those with disabilities, agoraphobia, anxiety, or severe depression, as beneficiaries of this accessibility, a finding echoed elsewhere in the literature on digital solutions to treatment gaps in mental health (Ebert et al., 2018). For some practitioners, this extended reach is itself an affordance, providing them with a sense of pride and mission: "I think that's what motivated me to keep going, that I knew that I was helping people who would not have sought out a therapist in that area."

The affordance of accessibility also affords meaningful access for therapists by allowing them to overcome their own constraints. One practitioner from a rural area explained that her turn to teletherapy was motivated by the limitation of geography:
I'm used to a much more diverse population, and so it gives me the opportunity to work with lots of different kinds of people that I wouldn't otherwise be able to work with in my private practice office. So I really like that a lot. I get to work with people from all over the state instead of just people that live in my town.

For both clients and practitioners, DTCTMH lowers barriers to participation.

Accessibility is both an affordance and a constraint for practitioners. Platform advertising which conveys the 24-hr availability of therapeutic interaction- "Your therapist is waiting to chat with you right now" or "No scheduling needed"-makes practitioners responsible to set boundaries on their availability to respond. Practitioners described the effort to reeducate clients as routine:

I do try to help them understand that while they're welcome to message me 24 hours a day, that does not mean that I'm accessible necessarily 24 hours a day, because that seems to be a common misunderstanding that people are coming with.

Accessibility is not accidental-it is a business strategy bundled in excellent design. Forprofit platforms design their services to engage the greatest number of paying customers, 
and the friction-less journey from clicking on a webpage to talking with a therapist glosses over core components of ethical treatment, like informed consent, or the specifics of data collection by platforms. Multiple practitioners articulated concern that the incentive of a free trial week, a periodic promotion, and platform taglines like "Feeling better starts with a single message" misrepresent the work required to achieve meaningful mental health gains. These practitioners work with the awareness that their own ethics do not always coincide with the ethos of the platform that employs them, their work motivated by the potential to offer support to people who seek help. Therapists describe a constant responsibility to communicate to clients, especially those who are new to therapy, that the path to feeling better is not simple or swift. "The clients I get from (PLATFORM), yeah, they want instantaneous constant contact, and it's unrealistic and it's unhealthy." Some articulated the opinion that the affordance of accessibility — so critical to clients and to platforms - could actually be counterproductive to a treatment relationship, that a program of "Unlimited Messaging Ther-

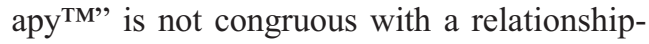
based change process which ideally models the delay of gratification, clear boundaries, and the habit of reflection. As a practitioner summed up,

you know, the immediate gratification mindset, I think that that's a big problem in our culture in general. And I think in providing therapy and therapeutic services that it's my ... it's my personal feeling, it's my job [Laughs] to discourage a lot of that thinking, immediate gratification and multi-tasking.

Anyone with access to the internet can try DTCTMH, and this accessibility obligates practitioners to delineate between clients who can benefit from virtual therapeutic interaction and those who require higher levels of care, including face-to-face therapy, medication, or in-patient services. Many interviewees referred to "appropriate" clients, and most reported a self-imposed protocol of refusing to partner with those clients whose needs seem too urgent to be addressed remotely, recommending the most distressed clients to seek face-to-face therapy or medication. Practitioners cultivate attention to "clues" that might indicate a client is at high risk, as one practitioner said, "I weed out people that ... that I feel like would be a concern." Affordance theory insists that affordances are specific relationships, dependent on the action capabilities of the perceiving animal. According to practitioners, platform-based virtual therapy bears out this model: the benefit it can afford depends entirely on the specifics of client need and capacity.

\section{Anonymity}

According to Bucher and Helmond (2016), technology affordances present the material qualities of technology as constitutive-in part — of sociality and communicative actions. The fact that virtual platform-based therapy is accessed via a screen and a keyboard, spatially remote from a provider, has critical implications for the interaction that ensues. These material constituents of virtual therapy afford the potential for clients to remain anonymous. Although on many platforms clients submit personal identifying information to the platform at registration for use in the case of emergency, a client can withhold any identifying information from their therapist. In the words of one practitioner, "I mean they're completely anonymous, like I'm not even sure if the names are accurate." However, clients see the names of their practitioners when electing a provider, and, while communication with a therapist is confined to the platform dashboard, "they have my full name so they could easily look me up and find out where my practice is or whatever."

Therapists are very clear that anonymity attracts clients to the modality, and that it increases client comfort. One therapist attested, "having that anonymity seems to work for them and gives them a safe space to share something that they might not if they were seeing somebody face-to-face, it gives them a little liberty." Anonymity, given this magnetism, is an affordance for the platform, 
reducing barriers to those seeking services. While many hesitate to endorse anonymity entirely, therapists describe themselves as inspired by the way in which the anonymity of DTCTMH affords the ability to reachand to engage - clients they would not otherwise serve. Anonymity, according to multiple practitioners, is an affordance which-like that of accessibility-allows them to serve clients who are uninterested in face-to-face therapy and who would be reluctant or unwilling to seek in person services, a phenomenon articulated elsewhere in the literature on therapy in virtual space (Ebert et al., 2018; Ehrlich, 2019) Two practitioners voiced a sentiment shared by many among the sample, one reporting, "there's a whole group of people that are just absolutely never going to set foot in an office and be forthcoming about what their situation is," whereas another attests, "anonymity makes people feel more confident. So, yeah, I think you do get a different client, you get somebody who wouldn't normally seek it out." Some diagnoses, multiple practitioners asserted, are especially well-served by the anonymity of TMH: especially diagnosis associated with shame or fear of discovery, as one said, "shame can be such a deterrent, right, for speaking out." Many therapists concur that anonymity speeds disclosure:

when it's totally anonymous and they're not looking at me and I'm not looking at them they tell me stuff, you know a lot of times people told me stuff that ... and you know they would say, "I've never told anyone this," and they would tell me almost right away because it felt safe.

Some platforms offer clients the option of video sessions, and the experience of being seen on video dilutes this effect, even under anonymous conditions: "But text and audio clients, well they will just tell you the darndest thing outright, you know, without hardly any rapport building at all, I suppose because it's so anonymous." This therapist, like many, reported, "progress can be a lot faster, because they got to the core of the issues of why they came to therapy much quicker."
Hutchby and Barnett (2005) specify that the "communicative affordances" associated with advancing technologies of interaction are relational, that they "may be different for one species than for another" (p. 151). While the platform feature of anonymity affords clients greater access to and freedom of communication, it acts as both affordance and constraint for practitioners. Some practitioner concerns are practical: anonymity makes it easier for a client to back out of therapy, and even clients who stay engaged may feel less of a sense of obligation to the therapist or to the process. Although no interviewee reported any such incident, a therapist cannot be sure that they are talking to the client, rather than to a friend, stranger, or partner who may not be entitled to the privileged therapeutic dialogue. As one practitioner said, "I never had, that I knew, like a switcheroo, or, who knows if an abusive husband or a girlfriend couldn't hop on, you know, and start talking to you as the person, how would you know?"

But for many practitioners, anonymity presents a constraint in the form of an ethical quandary: How can they do their job, and realize their obligation to prevent harm, if they do not know who a client is or where they are located? Although, according to many, the platform on which they work does have a protocol to supersede client-elected anonymity in cases of elevated risk, few trust the platform to step in and help them problem solve. One therapist said of clients, "I don't know where they live, I don't know how to get a hold of them outside of that platform. And I don't really trust the platform if there were some sort of emergency or something." Practitioners underline that, outside of emergency, they can only access identifying information through dialogue with a client. Across modalities, therapists who practice virtually are concerned by and attentive to the ethical dimension of this evolving practice (Békés \& Aafjes-van Doorn, 2020; Trub \& Magaldi, 2017), and practitioners interviewed for this study describe the effort of ethical practice within the corporate frame of a for-profit platform. Some therapists have gone to extraordinary lengths to help anonymous clients in 
distress, piecing together information to triangulate their client's location to call for a safety check, but anonymity constrains the ability to procure on the ground services for clients in crisis. As a constraint on ethical practice, anonymity has motivated some practitioners to create their own protocols for obtaining identifying information from clients as their own inflexible condition of service. As this practitioner reported, "I wound up creating my own informed consent where I would ask for their contact information and emergency contact information, and then I felt more comfortable."

\section{Practitioner-Specific Affordances: Meaning, Extended Reach, Learning, and Autonomy}

Social workers are challenged to build sustainable careers and to discover meaningful work in a landscape where service models are being forcibly evolved by the imperatives of profit and efficiency (Abramovitz \& Zelnick, 2018). Although they offer critique of the forprofit platforms to whom they contract, many therapists describe DTCTMH as meaningful work that affords a route to reaching new client populations, to professional autonomy, to ongoing learning, and to a functional balance between work and life. DTCTMH is opt-in, and those therapists who elect to continue this form of virtual practice remain because of the affordance or possibility to realize meaningful work. One form of meaning is a sense of efficacy, or the sense of providing client benefit. For some practitioners, client benefit is associated with the affordances of accessibility and anonymity:

I think that it's very effective for certain kinds of difficulties, certain kind of problems, I think it can be sort of very meaningful, I think people can become ... I think people really sort of like the idea that they can have this much access.

Another practitioner reported that, for her clients, anonymity accelerates benefit, satisfying her own professional desire to help: "I really enjoy it for that, you know because people feel better quicker because they just sort of unburden themselves, you know?" Literature that interrogates how therapists experience other forms of virtual practice echoes this finding: Practitioners describe themselves as emotionally connected and authentic in virtual space; they feel competent and able to forge an effective working alliance with clients (Békés \& Aafjes-van Doorn, 2020; Hanley \& Reynolds, 2009). Therapists in this study who work on platforms endorse the platform's utility for clients, even as they simultaneously practice face-to-face. One practitioner stated,

I don't want to give it up, because I now have started working face-to-face. I still want to have this. I feel connected with my client base that I have, I still feel that I'm doing good work, and I find I think there's value in it.

Another ascribed his sense of meaning to how DTCTMH can realize values of social work: "I would say like on a social justice or empowerment perspective, it can help marginalized clients in other countries as well as clients in rural areas where they can't really access care on a regular basis."

Telehealth affords practitioners an explicit awareness of expanded reach. Many practitioners derived a sense of purpose from providing service to new populations:

I think someone that might not seek face-toface counseling might not ever get any kind of help, and this is an opportunity and a forum where they could have more privacy, and I think that is an advantage of it, that people might access it that may have never accessed help.

Multiple practitioners reported interacting with new types of clients, especially those wishing to address experiences of stigmatized identity. Clients who are negotiating gender identity, infertility, or sexual orientation find platforms especially attractive. In this study, as in other research on TMH, working virtually attracts higher preponderance of clients experiencing diagnoses or situations associated with shame (Ehrlich, 2019): 
you know I've had people who were you know pedophiles, the ones who had never acted on it, but they had, you know, the motivation, and so controlling it was an issue, or whatever; they're never gonna go to anybody in a face-to-face situation, they'd be afraid to, and probably rightly so. So I think that's . . you know it serves people who would never . . . it could serve people, you know all kinds of people, but it's particularly valuable who would never go to face-to-face.

Practitioners appreciate the extended reach of virtual therapy: the literal ability to "meet clients where they are." One therapist specifically identified this reach as a meaningful affordance:

So it reaches people who like I said cannot go the traditional route. Either they can't afford it, or they don't have the time that they really need for someone if they're listening to them. And I think that's the beauty of it.

Social workers are mandated to lifelong learning, and some describe DTCTMH as a supportive mechanism to grow their professional capacity. Some use platforms to transition fields of practice, or to refine skills:

At my full-time job, I'm a supervisor, so I don't have any direct client contact, I just like review charts and staff, clients, and things like that with the clinicians that I supervise. So, I kind of wanted to try to do something more clinical, like after hours, for myself.

Others credit their platform-based work with deepening, even "transforming" their practice:

Those interventions over the last two years have all been morphed into tool kits that I give people that come in face-to-face, they have transformed into sort of writing, I have like a magazine that's coming out and a book, and none of those things would have ever developed had I not started working with people online. So, it has really transformed my practice, and taken it from sort of a basic psychotherapy approach to a skillbased approach.
One practitioner reported that, for those who prioritize autonomy, TMH - on or off a platform-affords the opportunity to "practice in a way that reflects your own passion and personality."

For therapists, platforms offer a means to earn income by deploying their professional skills, working flexibly with few overhead costs. A participant shared,

I mean I'll be completely honest, I don't have to hire a sitter, and I don't have to ask family to watch my kids, like for the most part that has been the convenience, I'm not spending money on office expense, and that sort of thing.

As another said, "You get to set your schedule and you get to work on your own time, and dedicate, you know, 40 forty hours a week, or 4 hours a week, or you know 1 hour a week." Extra income was important to many practitioners, enabling them to realize personal goals, from cultivating skills, to saving money, to keeping a beloved but low-paying job in social work:

I've worked at a full-time job, at a nursing home, in a (LOCATION), small little nursing home, which I loved, for nine years, and it doesn't pay the bills, and it's not enough. But I love it, I live basically next-door to where I work, I don't want to leave here, but I needed more money. And for years, or for quite a long time I was just in my mind was thinking, 'God, wouldn't it be so great if I could find, or if there was available, some sort of utilization for my social work, like counseling or whatever, that I could do on-line, you know after hours, on the weekends; I wouldn't have to travel or ... and then, you know, it evolved.

Many reported that the flexibility of platformbased work facilitated transition in their lives: embarking on a practice, leaving an unsatisfying job, the birth of a child or parenting, or managing retirement.

I started because I was trying to build my practice and it was just a nice little extra income 
that I could do while I was waiting for real people to walk in my door, but I really liked it.

The majority of this sample, like the majority of social workers, is female, and the affordance of flexibility emerged as a theme among practitioners seeking to balance family and the demands of professional life:

my kids could be winding down for the night, I can log onto my computer and I can read my messages and reply, so it's very easy to engage in this platform you know without really interfering with a lot of other things in my life.

\section{Limitations}

This study intentionally sought reflections, thoughts, and ideas about DTCTMH from licensed professional social workers to gain a more nuanced understanding of the practice of therapy in digital spaces. The primary limitations of this study are the generalizability of the findings; the small, selfselected sample of practitioners who choose to remain in practice on a DTC mental health platform; and a qualitative coding scheme. While the modalities may overlap, contract work on a platform may not be generalizable to all virtual therapy.

\section{Implications for the Field and Future Directions}

Whether they are pushed to adapt to virtual practice by market reform, institutional costcutting, by client demands for novel conduits to service or by their own curiosity and desire to provide services, many practitioners will be compelled to engage in new forms of practice (Glueckauf et al., 2018), and the platforms described here provide one point of entry, an opt-in apprenticeship in the mechanics on providing therapy digitally. Indeed, the NASW, ASWB, CSWE, \& CSWA Standards for Technology in Social Work Practice articulate the responsibility of social workers to understand technology mediated practice and its ethical applications.
Although little literature describes DTCTMH, the themes brought forward in this study reflect those at play in an evolving literature, which asks if TMH can provide an effective strategy to address the needs of clients. Research into the potential of TMH has long focused on "functional equivalence," or the congruence of virtual therapy to established, face-to-face forms of practice (Trub \& Magaldi, 2017). The lens of affordances used here focuses instead on what actions are made possible by the treatment under study-distilling interviews with practitioners to document some of the modality's unique potentials and constraints. The affordances lens reveals some of the attributes of DTCTMH that empower action: accessibility to clients and providers, anonymity, meaningful work, flexibility, autonomy, extended reach, and lifelong learning.

While the research that accompanied a first generation of virtual mental health interventions necessarily focused on documenting outcomes, more recent study delves into the complexities of therapy in this space-the lived thinking and feeling that accompanies the process of providing therapy to a client you may not be able to see (Békés \& Aafjes-van Doorn, 2020; Trub \& Magaldi, 2017). The practitioners in this study describe affordances that are multivalent: accessibility attracts new type of clients to therapy and may misrepresent the hard labor of making change; anonymity frees clients to be truthful, and perhaps also to abandon treatment more easily, In the specific example of therapeutic services marketed and mediated by a platform, the profit imperative structures services according to an alternate logic. This escalates pressure on practitioners to remain vigilant to the ethical dimension of their work, yielding supplemental consent protocols, strict rules about which clients to take on, and extraordinary interventions on behalf of clients in crisis. The practitioners who work on platforms operate actively aware of the tension between the opportunity they have to conduct meaningful work with an expanded population of clients and an employment structure built to maximize volume, 
reduce friction, minimize costs, and maximize earning.

Affordances are most visible in their uptake, when the possibility of action is realized. Thus, a digital platform's affordance of accessibility can be most simply cataloged by charting client use of services. The affordances identified here - and what they make available to both clients and practitionersoffer important description of a mental health landscape that cannot meet the needs of providers or clients without novel conduits to service. Long committed to "meeting clients where they are," all social workers have a duty to consider which forms of communication are most accessible to and practical for the clients they serve. In an accelerated technological culture, digital channels are the conduits of relationship and dialogue, and social workers must cultivate the skills of virtual outreach and connection. Multiple factors-including cost, geography, disability, the onus of travel, and the imperative of paid work-preclude access to and participation in activities that support mental health for many clients and communities, and TMH can solve these barriers. This constellation of mental health delivery channels can render therapy relevant to greater number of people: the practitioners interviewed here together assert that, as facilitated by DTCTMH platforms, these tools show promise in reaching new populations, that they can erode the limitations of ability and apprehension that preclude some individuals from seeking services. The NASW Code of Ethics charges social workers to challenge social injustice by "striv(ing) to ensure access to needed information, services, and resources," and TMH, realized ethically, is a practical tool to realize the "equality of opportunity."

Yet social workers who practice virtually forego the support of existing systems and structures. There is a fundamental mismatch between standard social work training and the realities of virtual practice, which forces practitioners to investigate new strategies and solutions - both for themselves and for their virtual clients. The existing structure of licensure fails to match the realities of service need or provision, frustrating those who might seek or provide care virtually. While TMH is not a new strategy of care, many practitioners describe platform-based therapy as a practice which requires them to seek training, do research, realize ethical guidelines, and to develop communities of practice on their own, outside of established channels. As one practitioner summed up, "It's kind of on us as the therapist." The formal mechanisms of the profession must evolve to meet a changing reality. Consistent with demand from the field, as articulated in the Council on Social Work Education's Futures Task Force (2018) report, as well as the NASW Code of Ethics's call for competence and lifelong learning, those who structure, design, and execute professional training must work to create and deliver content relevant for digital TMH. Social workers require training to acquire skills, licensure that testifies to their competence, and structural support to assure their longevity in the profession.

The use of affordances here is designed to provide analysis which augments comparison with a focus on those attributes of DTCTMH that enable action, articulating salient potentials of platform-based therapy. As virtual interventions to support mental health are increasingly documented by research, it will be important to focus on how and by whom these affordances are realized, and to examine the mechanisms of dose and specific modality. It will be critical to examine the particulars of the extended reach to new populations described here to understand, and to advance, social work's ability to serve marginalized groups. The practitioners interviewed here continue to practice virtually because they believe that - for a subset of individuals seeking treatment-platform-based therapy may afford accessible interpersonal interaction that supports mental health.

\section{Declaration of Conflicting Interests}

The author(s) declared no potential conflicts of interest with respect to the research, authorship, and/or publication of this article. 


\section{Funding}

The author(s) received no financial support for the research, authorship, and/or publication of this article.

\section{ORCID iD}

Lauri Goldkind (iD https://orcid.org/0000-00020967-3960

\section{References}

Abramovitz, M., \& Zelnick, J. (2018). Business as usual? A wake-up call for the human services. Graduate School of Social Work, Tuoro College; Silberman School of Social Work, Hunter College.

Andrews, G., Cuijpers, P., Craske, M. G., McEvoy, P., \& Titov, N. (2010). Computer therapy for the anxiety and depressive disorders is effective, acceptable and practical health care: A meta-analysis. PLOS ONE, 5(10), Article e13196.

Barak, A., \& Grohol, J. M. (2011). Current and future trends in internet-supported mental health interventions. Journal of Technology in Human Services, 29(3), 155-196.

Békés, V., \& Aafjes-van Doorn, K. (2020). Psychotherapists' attitudes toward online therapy during the COVID-19 pandemic. Journal of Psychotherapy Integration, 30(2), 238-247.

Berrouiguet, S., Baca-García, E., Brandt, S., Walter, M., \& Courtet, P. (2016). Fundamentals for future mobile-health (mHealth): A systematic review of mobile phone and web-based text messaging in mental health. Journal of Medical Internet Research, 18(6), Article e135.

Birt, L., Scott, S., Cavers, D., Campbell, C., \& Walter, F. (2016). Member checking: A tool to enhance trustworthiness or merely a nod to validation? Qualitative Health Research, 26(13), 1802-1811.

Bucher, T., \& Helmond, A. (2016). Social media affordances and interfaces. In J. Burgess, T. Poell, \& A. Marwick (Eds.), Sage handbook of social media (pp. 1164-1180). SAGE.

Chan, S., Li, L., Torous, J., Gratzer, D., \& Yellowlees, P. M. (2018). Review of use of asynchronous technologies incorporated in mental health care. Current Psychiatry Reports, 20(10), 85.

Colbow, A. J. (2013). Looking to the future: Integrating tele-mental health therapy into psychologist training. Training and Education in Professional Psychology, 7(3), 155-165.

Corbin, J. M., \& Strauss, A. (1990). Grounded theory research: Procedures, canons, and evaluative criteria. Qualitative Sociology, 13(1), 3-21.

Ebert, D. D., Van Daele, T., Nordgreen, T., Karekla, M., Compare, A., Zarbo, C., ... . Kaehlke, F. (2018). Internet-and mobile-based psychological interventions: Applications, efficacy, and potential for improving mental health. European Psychologist, 23, 167-187.

Ehrlich, L. T. (2019). Teleanalysis: Slippery slope or rich opportunity? Journal of the American Psychoanalytic Association, 67(2), 249-279.

Figueroa, C. A., \& Aguilera, A. (2020). The need for a mental health technology revolution in the COVID-19 pandemic. Frontiers in Psychiatry, 11, Article 523.

Firth, J., Torous, J., Nicholas, J., Carney, R., Rosenbaum, S., \& Sarris, J. (2017). Can smartphone mental health interventions reduce symptoms of anxiety? A meta-analysis of randomized controlled trials. Journal of Affective Disorders, 218, 15-22.

Gaver, W. W. (1991). Technology affordances. In Proceedings of the SIGCHI conference on human factors in computing systems (pp. 7984). Association for Computing Machinery. http://dl.acm.org/citation.cfm?id=108856

Gaver, W. W. (1996). Situating action II: Affordances for interaction: The social is material for design. Ecological Psychology, 8(2), 111-129.

Gibson, J. J. (2015). The ecological approach to visual perception. Classic editions. Psychology Press.

Glueckauf, R. L., Maheu, M. M., Drude, K. P., Wells, B. A., Wang, Y., Gustafson, D. J., \& Nelson, E. L. (2018). Survey of psychologists' telebehavioral health practices: Technology use, ethical issues, and training needs. Professional Psychology: Research and Practice, 49(3), 205-219.

Godleski, L., Darkins, A., \& Peters, J. (2012). Outcomes of 98,609 US Department of Veterans Affairs patients enrolled in telemental health services, 2006-2010. Psychiatric Services, 63(4), 383-385.

Goldkind, L., \& Wolf, L. (2020). Selling your soul on the information superhighway: Consenting to services in direct-to-consumer tele-mental health. Families in Society, 101(1), 6-20. 
Haig-Ferguson, A., Loades, M., Whittle, C., Read, R., Higson-Sweeney, N., Beasant, L., ... Crawley, E. (2019). "It's not one size fits all"; the use of videoconferencing for delivering therapy in a Specialist Paediatric Chronic Fatigue Service. Internet Interventions, 15, 43-51.

Hanley, T., \& Reynolds, D. (2009). Counselling psychology and the internet: A review of the quantitative research into online outcomes and alliances within text-based therapy. Counselling Psychology Review, 24(2), 4-13.

Hilty, D. M., Ferrer, D. C., Parish, M. B., Johnston, B., Callahan, E. J., \& Yellowlees, P. M. (2013). The effectiveness of telemental health: A 2013 review. Telemedicine and e-Health, 19(6), 444-454.

Hood, R. (2015). How professionals experience complexity: An interpretative phenomenological analysis. Child Abuse Review, 24(2), $140-152$.

Hutchby, I. (2001). Conversation and technology: From the telephone to the internet. Polity Press.

Hutchby, I., \& Barnett, S. (2005). Aspects of the sequential organization of mobile phone conversation. Discourse Studies, 7(2), 147-171.

Karyotaki, E., Riper, H., Twisk, J., Hoogendoorn, A., Kleiboer, A., Mira, A., . . . Andersson, G. (2017). Efficacy of self-guided internet-based cognitive behavioral therapy in the treatment of depressive symptoms: A meta-analysis of individual participant data. JAMA Psychiatry, 74(4), 351-359.

Langarizadeh, M., Tabatabaei, M. S., Tavakol, K., Naghipour, M., Rostami, A., \& Moghbeli, F. (2017). Telemental health care, an effective alternative to conventional mental care: A systematic review. Acta Informatica Medica, 25(4), 240-246.

Luxton, D. D., Pruitt, L. D., \& Osenbach, J. E. (2014). Best practices for remote psychological assessment via telehealth technologies. Professional Psychology: Research and Practice, 45(1), 27-35.

Malhotra, S., Chakrabarti, S., \& Shah, R. (2013). Tele psychiatry: Promise, potential, and challenges. Indian Journal of Psychiatry, 55(1), 3-11.

Mental Illness. (2019). https://www.nimh.nih.gov/ health/statistics/mental-illness.shtml

Neary, M., \& Schueller, S. M. (2018). State of the field of mental health apps. Cognitive and Behavioral Practice, 25(4), 531-537.
Padgett, D. K. (1998). Does the glove really fit? Qualitative research and clinical social work practice. Social Work, 43(4), 373-381.

Payne, L., Flannery, H., Kambakara Gedara, C., Daniilidi, X., Hitchcock, M., Lambert, D., Taylor, C., \& Christie, D. (2020). Business as usual? Psychological support at a distance. Clinical Child Psychology and Psychiatry, 25(3), 672-686. https://doi. org/10.1177/1359104520937378

Postigo, H. (2016). The socio-technical architecture of digital labor: Converting play into YouTube money. New Media \& Society, 18(2), 332-349.

Rebello, T. J., Marques, A., Gureje, O., \& Pike, K. M. (2014). Innovative strategies for closing the mental health treatment gap globally. Current Opinion in Psychiatry, 27(4), 308314.

Sabin, J. E., \& Skimming, K. (2015). A framework of ethics for telepsychiatry practice. International Review of Psychiatry, 27, 490495.

Salsberg, E., Quigley, L., Mehfoud, N., Acquaviva, K. D., Wyche, K., \& Silwa, S. (2017). Profile of the social work workforce. https:// hsrc.himmelfarb.gwu.edu/cgi/viewcontent. cgi ?article $=1015 \&$ context $=$ sphhs_policy_ workforce_facpubs

Smith, J. A., Flowers, P., \& Larkin, M. (2009). Interpretative phenomenological analysis: Theory, method and research. SAGE.

Trub, L., \& Magaldi, D. (2017). Left to our own devices. Psychoanalytic Perspectives, 14(2), 219-236.

Turgoose, D., Ashwick, R., \& Murphy, D. (2018). Systematic review of lessons learned from delivering tele-therapy to veterans with post-traumatic stress disorder. Journal of Telemedicine and Telecare, 24(9), 575-585.

Varker, T., Brand, R. M., Ward, J., Terhaag, S., \& Phelps, A. (2019). Efficacy of synchronous telepsychology interventions for people with anxiety, depression, posttraumatic stress disorder, and adjustment disorder: A rapid evidence assessment. Psychological Services, 16(4), 621-635.

Vernig, P. M. (2016). Tele-mental health: Digital disruption and the opportunity to expand care. Journal of the American Psychiatric Nurses Association, 22(1), 73-75.

Watts, S., Marchand, A., Bouchard, S., Gosselin, P., Langlois, F., Belleville, G., \& Dugas, M. 
J. (2020). Telepsychotherapy for generalized anxiety disorder: Impact on the working alliance. Journal of Psychotherapy Integration, 30(2), 208-225.

Wellman, B., Quan-Haase, A., Boase, J., Chen, W., Hampton, K., Díaz, I., \& Miyata, K. (2003). The social affordances of the internet for networked individualism. Journal of Computer-Mediated Communication, 8(3),
JCMC834. http://onlinelibrary.wiley.com/ doi/10.1111/j.1083-6101.2003.tb00216.x/ abstract

Wilkerson, D. A., Wolfe-Taylor, S. N., Deck, C. K., Wahler, E. A., \& Davis, T. S. (2020). Telebehavioral practice basics for social worker educators and clinicians responding to COVID-19. Social Work Education, 39(8), 1137-1145. 